\title{
Global Solutions to the Initial Value Problem for the Nonlinear Boltzmann Equation
}

By

Takaaki NISHIDA* and Kazuo IMAI*

\begin{abstract}
The nonlinear Boltzmann equation in the rarefied gas dynamics is investigated for the gas molecules with the cut-off hard potential in the sense of Grad. The solution to the initial value problem is proved to exist uniquely in the large in time and to have the decay order of $(1+t)^{-8 / 4}$ as $t \rightarrow+\infty$ for the small initial data in the space $H_{3, s} \cap L^{1}\left(x ; L^{2}(v)\right)$. The decay order is improved to $(1+t)^{-5 / 4}$ by the additional assumptions on the initial data.
\end{abstract}

\section{$\S$ 1. Introduction}

The nonlinear Boltzmann equation in the rarefied gas dynamics for the gas molecules with the cut-off hard potential in the sense of Grad is given around the absolute Maxwellian state $M=(2 \pi)^{-3 / 2} \exp \left(-|v|^{2} / 2\right)$ by the following: (cf. [2], [3])

$$
\begin{aligned}
\partial f(t, x, v) / \partial t+\sum_{j=1}^{3} v_{j} \partial f(t, x, v) / \partial x_{j}=L f+\nu \Gamma(f, f) \\
\text { in } t \geqq 0, x \in R^{3}, v \in R^{3} . \\
f(0, x, v)=f_{0}(x, v) \text { in } x \in R^{3}, v \in R^{3} .
\end{aligned}
$$

Here the operator $L$ acting only on $v$ is linear and nonpositive, i.e.,

$$
\begin{aligned}
& (L f, f) \leqq 0 \text { for } f \in D(L) \text { and } \\
& L f=0 \text { iff } f \in\left\{\psi_{j}\right\}_{j=1}^{5}=\left\{M^{1 / 2}, v_{j} M^{1 / 2},|v|^{2} M^{1 / 2}\right\}
\end{aligned}
$$

and it is decomposed as follows:

$$
L f=-\nu(v) f+K f \quad \text { in } \quad L^{2}(v),
$$

Communicated by S. Matsuura, December 15, 1975.

* Department of Applied Mathematics and Physics, Kyoto University, Kyoto. 
where $\nu(v)$ is a monotone function in $|v|$ and

$$
0<\nu_{0} \leqq \nu(v) \leqq \nu_{1}(1+|v|) \text {, and }
$$

$K$ is a compact selfadjoint operator on $L^{2}(v)$.

The operator $\nu \Gamma(f, g)$ depends on $v$ and is bilinear in $f, g$, and the further properties are stated later.

The initial value problem (1.1) (1.2) is considered by Grad [3], [4] locally in time, and by Ukai [12] in the large in time for the small initial data which are periodic in $x$, whose solution decays exponentially in $t$. Here we consider the pure initial value problem (1.1) (1.2) and prove that the solution exists uniquely in the large in time for the initial data $f_{0}(x, v) \in H_{3,3} \cap L^{1}\left(x ; L^{2}(v)\right)$ with the small norm in these spaces and that it decays with the order of $(1+t)^{-3 / 4}$ as $t \rightarrow+\infty$, where

$$
H_{l, m}=\left\{f=f(x, v) ;\|f\|_{l, m} \equiv \sup _{v}(1+|v|)^{m}\|f(\cdot, v)\|_{H^{l}}<+\infty\right\}
$$

(1.7) $\quad H^{l}$ is the Sobolev space of $L^{2}$-functions in $x$ with the $l$-th $L^{2}$ derivatives, and

$(1 \cdot 8) \quad L^{p}\left(x ; L^{2}(v)\right)$ is the $L^{p}$-space in $x$ with the value in $L^{2}(v)$ and

$$
\|f\|_{L^{p}\left(x ; L^{2}(v)\right)}^{p}=\int\|f(x, \cdot)\|_{L^{(v)}}^{p} d x .
$$

The additional conditions on the initial data such that

$$
x f_{0}(x, v) \in L^{1}\left(x ; L^{2}(v)\right) \text { and } \iint f_{0}(x, v) \psi_{j}(v) d x d v=0, j=1,2, \cdots, 5
$$

improve the decay order to $(1+t)^{-5 / 4}$ as $t \rightarrow+\infty$. In order to get the existence theorem and asymptotic decay of solutions, we use the arguments in [3], [12] cited above and those in [1] where the linearized Boltzmann equation is discussed about the fluid dynamical approximation, and those in [5], where the decay of solutions in the whole space as $t \rightarrow+\infty$ is considered for the Broadwell model of Boltzmann equation.

Acknowledgement: The authors would like to thank Mr. K. Inoue and Prof. Y. Ohya for the original discussions on this work and to thank Prof. J. Glimm for his hearty encouragement to this work. 


\section{$\S$ 2. Linearized Boltzmann Equation}

We summarize here some known results about the linearized Boltzmann equation,

$$
\partial f(t, x, v) / \partial t+v \cdot \partial f / \partial x=L f .
$$

It is considered in the Fourier transform in $x$ :

$$
\begin{gathered}
\partial \widehat{f}(t, k, v) / \partial t=-i k \cdot v \widehat{f}+L \widehat{f} \equiv B \widehat{f} \\
\text { in } t \geqq 0, k \in R^{3}, v \in R^{3} .
\end{gathered}
$$

Let $k \in R^{3}$ be a fixed parameter and consider the following operators in $L^{2}(v)$.

$$
\begin{aligned}
& B_{k}=-i k \cdot v-\nu(v)+K, \\
& A_{k}=-i k \cdot v-\nu(v),
\end{aligned}
$$

where $D\left(A_{k}\right)=D\left(B_{k}\right)=\left\{f \in L^{2}(v), A_{k} f \in L^{2}(v)\right\}$.

Since $K$ is a compact operator, $B_{k}$ is considered as the compact perturbation from $A_{k}$.

Proposition 1. $B_{k}$ generates the strongly continuous contraction semigroup $e^{t B_{k}}$ on $L^{2}(v)$.

Proof. See [1], [9].

Definition. $\sigma\left(B_{k}\right), \sigma_{e}\left(B_{k}\right)$ and $\sigma_{d}\left(B_{k}\right)$ denote the spectrum, essential spectrum in the sense of Browder [11] and the set of isolated eigenvalues with finite multiplicity, respectively.

Proposition 2. $\sigma_{e}\left(B_{k}\right) \subset \sigma_{e}\left(A_{k}\right) \cup\left\{\lambda_{l}=\beta+i \gamma, \beta \leqq-\nu(v), \gamma=-k \cdot v\right.$ for $\left.\forall v \in R^{3}\right\}, \sigma\left(B_{k}\right)=\sigma_{e}\left(B_{k}\right) \cup \sigma_{d}\left(B_{k}\right)$.

Proof. See [1], [8].

It is wellknown (cf. [2]) that $0 \in \sigma\left(B_{0}\right)=\sigma(L)$ is an eigenvalue with multiplicity 5 and its eigenspace is spanned by $\left\{\psi_{j}\right\}_{j=1}^{5}$, and that 
the other eigenvalue of $B_{0}$ (if exist) are on the negative real axis such that $0>\lambda_{1} \geqq \lambda_{2} \geqq \cdots \rightarrow-\nu_{0}$.

Now let us parametize $k=\kappa \omega$ where $\kappa \in R^{1}, \omega \in S^{2}$.

Proposition 3. There exists $\delta>0, \beta_{1}>0$ such that for $|k|<\delta$

$$
\sigma_{d}\left(B_{k}\right) \cap\left\{\operatorname{Re} \lambda>-\beta_{1}\right\}=\left\{\alpha_{j}(k)\right\}_{j=1}^{5},
$$

where the perturbed eigenvalues $\alpha_{j}(k)$ of 0 are smooth in $\kappa,|\kappa|<\delta$ and have the expansion

$$
\alpha_{j}(k)=a_{j, 1}(i \kappa)+a_{j, 2}(i \kappa)^{2}+a_{j, 3}(i \kappa)^{3}+0\left(\kappa^{4}\right),
$$

where

$$
a_{j, 2}>0, j=1,2, \cdots, 5 \text {. }
$$

The corresponding eigenfunctions are smooth in $\kappa$ and have the expansion

$$
e_{j}(k)=e_{j, 0}(\omega)+e_{j, 1}(\omega)(i \kappa)+e_{j, 2}(\omega)(i \kappa)^{2}+\cdots,
$$

and

$$
\left(e_{j}(-k), e_{l}(k)\right)_{L^{2}(v)}=\delta_{j, l}, j, l=1,2, \cdots, 5 \text {. }
$$

Proof. In particular for the hard sphere molecule, the expansions for $\alpha_{j}(k)$ and $e_{j}(k)$ converge in $\kappa,|\kappa|<\delta$ and they are analytic in $\kappa$ there. (cf. [7], [8]) For the general cut-off hardpotential, see [1].

\section{Proposition 4.}

(i) For any fixed $\beta<\nu_{0}$,

$$
\sup _{\substack{\operatorname{Re} \lambda \geq \beta \\|k|>r}}\left\|K\left(\lambda-A_{k}\right)^{-1}\right\| \rightarrow 0 \text { as } r \rightarrow+\infty \text {. }
$$

(ii) For any fixed $\beta<\nu_{0}$, any fixed $r>0$,

$$
\sup _{\substack{\operatorname{Re} \lambda \geq-\beta \\|k| \leq r \\|\lambda| \geq l}}\left\|K\left(\lambda-A_{k}\right)^{-1}\right\| \rightarrow 0 \text { as } l \rightarrow+\infty .
$$

(iii) For any fixed $\beta<\nu_{0}$, there are no eigenvalues of $B_{k}$ in $\{\operatorname{Re} \lambda \geqq-\beta\}$ for large $|k|$. And for $|k| \leqq \forall r<+\infty$, there exists $c=c(r)$ such that 


$$
\sigma_{d}\left(B_{k}\right) \cap\{\operatorname{Re} \lambda \geqq-\beta\} \subset\{|\operatorname{Im} \lambda| \leqq c\} .
$$

Proof. See [12]. (iii) follows from (i) and (ii).

If we note that the set $\sigma_{d}\left(B_{k}\right)$ in $\operatorname{Re} \lambda>-\beta\left(>-\nu_{0}\right)$ is continuous in $k \in R^{1}$ and so it is in $k \in R^{3}$ by using the rotation in $v$ for $B_{k}$, it follows from propositions 3 and 4 that for any $k,|k|>\delta>0$ there exists $\beta_{2}>0$ such that

$$
\sigma_{d}\left(B_{k}\right) \subset\left\{\operatorname{Re} \lambda<-\beta_{2}\right\} .
$$

Theorem 1. There exists $\delta>0, \beta_{1}>0, \beta_{2}>0$ such that the following (i) and (ii) are valid for any $f \in D\left(B_{k}\right)$.

(i) For any $k,|k| \leqq \delta$,

$$
\begin{aligned}
e^{t B_{k}} f= & \lim _{r \rightarrow+\infty} \frac{1}{2 \pi i} \int_{-\beta_{1}-i r}^{-\beta_{1}+i r} e^{t \lambda}\left(\lambda-B_{k}\right)^{-1} f d \lambda \\
& +\sum_{j=1}^{5} e^{t \alpha_{j}(k)}\left(e_{j}(-k), f\right)_{L^{2(v)}} e_{j}(k),
\end{aligned}
$$

where $\alpha_{j}(k)$ and $e_{j}(k)(j=1,2, \cdots, 5)$ are the eigenvalues and the corresponding eigenfunctions of $B_{k}$ in proposition 3.

(ii) For any $k,|k| \geqq \delta$,

$$
e^{t B_{k}} f=\lim _{r \rightarrow+\infty} \frac{1}{2 \pi i} \int_{-\beta_{2}-i r}^{-\beta_{2}+i r} e^{t \lambda}\left(\lambda-B_{k}\right)^{-1} f d \lambda .
$$

Proof. If we note that proposition 4 implies $\left\|\left(\lambda-B_{k}\right)^{-1} f\right\| \rightarrow 0$ as $|\operatorname{Im} \lambda| \rightarrow \infty$ for $\operatorname{Re} \lambda \geqq-\beta\left(>-\nu_{0}\right),[1]$ gives (i) and (2.5) gives (ii).

Remark The first terms on the right hand side in $(2 \cdot 6)$ and $(2 \cdot 7)$ can be rewriten in the form (cf. [12]);

$$
\begin{aligned}
& \lim _{r \rightarrow+\infty} \frac{1}{2 \pi i} \int_{-\beta_{j}-i r}^{-\beta_{j}+i r} e^{t \lambda}\left(\lambda-B_{k}\right)^{-1} f d \lambda \\
&=e^{t A_{k}} f+e^{-\beta_{j} t} \lim _{r \rightarrow+\infty} \frac{1}{2 \pi i} \int_{-i r}^{i r} e^{t \lambda}\left(\lambda-A_{k}\right)^{-1} \\
& \cdot\left(I-K\left(\lambda-A_{k}\right)^{-1}\right)^{-1} K\left(\lambda-A_{k}\right)^{-1} f d \lambda
\end{aligned}
$$




$$
=e^{t A_{k}} f+e^{-\beta_{j} t} Z\left(\beta_{j}, t\right) f,
$$

where $\left\|Z\left(\beta_{j}, t\right)\right\| \leqq c$ independent of $t . \quad(j=1,2)$

\section{$\S 3$. Decay of Solutions for the Linearized Boltzmann Equation}

Definition. $H=\left\{f(x, v) \in L^{2}(x, v),(x, v) \in R^{6}\right\} \subset L^{2}\left(x ; L^{2}(v)\right)$,

$$
\begin{aligned}
& \hat{H}=\left\{\widehat{f}(k, v)=(2 \pi)^{-3 / 2} \int e^{-i k \cdot x} f(x, v) d x \text { for } f \in H\right\} \\
& \quad \equiv L^{2}(k, v) \subset L^{2}\left(k ; L^{2}(v)\right), \\
& H_{l}=\left\{f \in H,|k|^{2} \widehat{f}(k, v) \in \hat{H}\right\}, \\
& \|f\|_{l^{2}}=\int(1+|k|)^{2 l}\|\widehat{f}(k, v)\|_{L^{2}(v)}^{2} d k .
\end{aligned}
$$

Definition. Boltzmann semigroup (cf. [1]).

$$
e^{t B} f=(2 \pi)^{-3 / 2} \int e^{i k \cdot x} e^{t B_{k}} \widehat{f}(k, v) d k \quad \text { for } \quad f(x, v) \in H .
$$

Theorem 2. The solution of the linearized Boltzmann equation $(2 \cdot 1)$ with the initial $f(x, v)$ has the following decay estimate for $l=0,1,2, \cdots$.

(i) Let $f(x, v) \in H_{l} \cap L^{1}\left(x ; L^{2}(v)\right)$. Then

$$
\left\|e^{t B} f\right\|_{l} \leqq c\left(\|f\|_{l}+\|f\|_{L^{1}\left(x ; L^{2}(v)\right)}\right) /(1+t)^{3 / 4} .
$$

(ii) In addition to the condition of (i) let $x f(x, v) \in L^{1}\left(x ; L^{2}(v)\right)$ and

$$
\iint \psi_{j}(v) f(x, v) d x d v=0 \text { for } \quad j=1,2, \cdots, 5 . \quad \text { Then }
$$

$$
\left\|e^{t B} f\right\|_{l} \leqq c\left(\|f\|_{l}+\|f\|_{L^{1}\left(x ; L^{2}(v)\right)}+\|x f\|_{L^{1}\left(x ; L^{2}(v)\right)}\right) /(1+t)^{5 / 4} .
$$

(iii) Let $f \in H_{l},\|\hat{f}(k, v)\|_{L^{2}(v)} \leqq c$ for any $k \in R^{3}$ and

$$
\int \psi_{j}(v) f(x, v) d v=0 \text { for } \quad \text { a.a. } x \in R^{3}, j=1,2, \cdots, 5 \text {. Then }
$$

$$
\left\|e^{t B} f\right\| \leqq c\left(\|f\|_{l}+\sup _{k}\|\widehat{f}(k, \cdot)\|_{L^{2}(v)}\right) /(1+t)^{5 / 4}
$$

It is shown in [10] that the decay of solution to $(2 \cdot 1)$ as $t \rightarrow+\infty$ 
without the decay order for the hard sphere molecule.

Proof. Let us estimate

$$
\begin{aligned}
\left\|e^{t B} f\right\|_{l^{2}} & =\int(1+|k|)^{2 t}\left\|e^{t B_{k}} f(k, v)\right\|_{L^{2}(v)}^{2} d k \\
& =\int_{|k| \leqq \delta}+\int_{|k|>\delta}=I+I_{1} .
\end{aligned}
$$

By Theorem 1 (ii) and the remark to it,

$$
I_{1} \leqq c^{2} e^{-2 \beta_{2} t} \int_{|k|>\delta}(1+|k|)^{2 l}\|\hat{f}(k, v)\|_{L^{2}(v)}^{2} d k
$$

For $|k| \leqq \delta$ we decompose $I$ as follows;

$$
I=\int_{|k| \leqq \delta} \leqq I_{2}+I_{3}
$$

where $I_{2}$ and $I_{3}$ correspond to the first term and the second term in the right hand side of $(2 \cdot 6)$ for $e^{t B_{k}} \hat{f}$ respectively. Therefore using again the remark to Theorem 1 we have

$$
\begin{aligned}
I_{2} & \leqq \int_{|k| \leqq \delta} c^{2} e^{-\beta_{1} t}(1+|k|)^{2 l}\|\widehat{f}(k, v)\|_{L^{2}(v)}^{2} d k \\
I_{3} & \leqq \int_{|k| \leqq \delta} c^{2}(1+|k|)^{2 l}\left\|\sum_{j=1}^{5} e^{t \alpha_{j}(k)}\left(e_{j}(-k), \hat{f}(k, \cdot)\right)_{L^{2}(v)} \mathcal{C}_{j}(k)\right\|_{L^{2}(v)}^{2 !} d k \\
& \leqq c^{2} \sup _{|k| \coprod^{\delta}}\|\hat{f}(k, \cdot)\|_{L^{2}(v)}^{2} \int_{|k| \leqq \delta} e^{-a|k|^{2 t}} d k \\
& \leqq c^{2}\|f\|_{L^{1}\left(x: L^{2}(v)\right)}^{2} /(1+t)^{3 / 2}, \quad\left(a=\min _{j} a_{j, 2}\right)
\end{aligned}
$$

Thus we have for $\beta_{0}=\min \left\{\beta_{1}, \beta_{2}\right\}$

$$
\begin{aligned}
& \left\|e^{t B} f\right\|_{l^{2}} \leqq I_{1}+I_{2}+I_{3} \\
& \quad \leqq c^{2} e^{-2 \beta_{0} t}\|f\|_{l}{ }^{2}+c^{2}\|f\|_{L^{1}\left(x ; L^{2}(v)\right)}^{2} /(1+t)^{3 / 2} .
\end{aligned}
$$

(ii) and (iii) are proved analogously. (cf. [5]). Q.E.D.

\section{$\S$ 4. Existence and Decay of Solutions for the Nonlinear Boltzmann Equation}

In order to get the existence and the decay estimate of the solutions to the Cauchy problem (1.1) (1.2) we follow the line of [3] and 
[12], when the space of functions $H_{l, m}$ for $l, m \geqq 3$ is used. (cf. (1.6))

Theorem 3. Let $l, m \geqq 3$

(i) Let the initial data $f_{0}(x, v) \in H_{l, m} \cap L^{1}\left(x ; L^{2}(v)\right)$ and put

$$
E_{1}=\left\|f_{0}\right\|_{l, m}+\int\left\|f_{0}(x, \cdot)\right\|_{L^{2}(v)} d x
$$

There exists a constant $E_{0}>0$ such that for any initial data with $E_{1}$ $<E_{0}$ the solution $f(t)$ of $(1 \cdot 1)(1 \cdot 2)$ exists uniquely in the large in time and has the decay estimate,

$$
\|f(t)\|_{l, m} \leqq c E_{1} /(1+t)^{3 / 4} .
$$

(ii) In addition let the initials satisfy $x f_{0}(x, v) \in L^{1}\left(x ; L^{2}(v)\right)$ and

$$
\iint \psi_{j}(v) f_{0}(x, v) d x d v=0, j=1,2, \cdots, 5
$$

Put $E_{2}=E_{1}+\int\left\|x f_{0}(x, v)\right\|_{L^{2}(v)} d x$.

Then the decay estimate of the solution of (i) improves to

$$
\|f(t)\|_{t, m} \leqq c E_{2} /(1+t)^{5 / 4} \text {. }
$$

Proof. In order to obtain the solution of $(1 \cdot 1),(1 \cdot 2)$ we introduce the iteration

$$
\begin{aligned}
& f^{n}(t)=e^{t B} f_{0}+\int_{0}^{t} e^{(t-s) B} \nu \Gamma\left(f^{n-1}(s), f^{n-1}(s)\right) d s, n=1,2, \cdots \\
& f^{0}(t)=0
\end{aligned}
$$

in $H_{l, m} \subset H_{l}$ where $e^{t B}$ is the linear solution operator in Theorem 2. To prove the convergence of this iteration globally in time, we use the integral equation

$$
f(t)=e^{t B} f_{0}+\int_{0}^{t} e^{(t-s) B} \nu g(s) d s
$$

where $\nu g(t)=\nu(v) g(t, x, v)$ is assumed to satisfy the conditions of Theorem 2, (iii), and follow Grad's and Ukai's arguments ([3], [12]). We use the operaters $e^{t A} f=e^{-t \nu(v)} f(x-t v, v)$ and a version of $(4 \cdot 2)$ 


$$
f(t)=e^{t A} f_{0}+\int_{0}^{t} e^{(t-s) A} \nu g(s) d s+\int_{0}^{t} e^{(t-s) A} K f(s) d s
$$

to estimate $(4 \cdot 2)$ in $H_{l, m}$. Using the property of $K$ ([2], [3])

has the next estimates for $\beta=3 / 4,5 / 4$

$$
\begin{aligned}
& \|f\|_{\beta}{ }^{l, 0} \leqq c\left(\left\|f_{0}\right\|_{l, 0}+\|g\|_{\beta}{ }^{l, 0}+\|f\|_{\beta}{ }^{l}\right) \\
& \|f\|_{\beta}{ }^{l, j} \leqq c\left(\left\|f_{0}\right\|_{l, j}+\|g\|_{\beta}{ }^{l, j}+\|f\|_{\beta}{ }^{l, j-1}\right), \quad j \geqq 1,
\end{aligned}
$$

where $c$ means some positive constant and

$$
\begin{gathered}
\|f\|_{\beta}{ }^{l, j} \equiv \sup _{t \geqq 0}(1+t)^{\beta}\|f(t)\|_{l, j}, \\
\|f\|_{\beta}{ }^{l} \equiv \sup _{t \geqq 0}(1+t)^{\beta}\|f(t)\|_{l} .
\end{gathered}
$$

Iterating these inequalities $j=0,1,2 \cdots m$, we obtain

$$
\|f\|_{\beta}^{l, m} \leqq c\left(\left\|f_{0}\right\|_{l, m}+\|g\|_{\beta}^{l, m}+\|f\|_{\beta}^{l}\right) .
$$

To estimate $\|f\|_{\beta}{ }^{l}$ we consider $(4 \cdot 2)$ in $H_{l}$. By the aid of the inequality

$$
(1+t)^{\beta} \int_{0}^{t} \frac{d s}{(1+s)^{2 \beta}\{1+(t-s)\}^{5 / 4}} \leqq c
$$

$c$ : independent of $t$,

Theorem 2 gives

$$
\|f\|_{\beta}{ }^{l} \leqq c\left(E+\|g\|_{2 \beta}^{l, m}+\sup _{\substack{k \in R^{3} \\ t \geqq^{3}}}(1+t)^{2 \beta}\left\|(\nu g)^{\wedge}\right\|_{L^{2}(v)}\right)
$$

where $E=E_{1}, E_{2}$ for $\beta=3 / 4,5 / 4$ respectively. From (4.6) and (4.8) it follows that

$$
\|f\|_{\beta}^{l, m} \leqq a E+b\left(\|g\|_{2^{2 \beta}}^{l, m}+\sup _{\substack{k \in R^{s} \\ t \geq 0}}(1+t)^{2 \beta}\left\|(\nu g)^{\wedge}\right\|_{L^{2}(v)}\right),
$$

$a, b:$ positive constants.

Now we can set $g(t)=\Gamma(h(t), h(t)), h \in H_{l, m}$. By virtue of [3] it has the estimates

$(4 \cdot 10) \quad\|\Gamma(h, h)\|_{2 \beta}^{l, m} \leqq c\left(\|h\|_{\beta}^{l, m}\right)^{2}$,

$(4 \cdot 11) \sup _{\substack{k \in R^{3} \\ t \geqq 0}}(1+t)^{2 \beta}\left\|(\nu \Gamma(h, h))^{\wedge}\right\|_{L^{2}(v)} \leqq c \sup _{t \geq 0}(1+t)^{2 \beta}\|\nu \Gamma(h, h)\|_{L^{2}\left(v ; L^{1}(x)\right)}$

$$
\leqq c\left(\|h\|_{\beta}^{0, m}\right)^{2} .
$$


Hence we obtain

$$
\|f\|_{\beta}^{l, m} \leqq a E+b c\left(\|h\|_{\beta}^{l, m}\right)^{2} .
$$

This inequality shows that there exists a constant $E_{0}$ such that for any $E<E_{0}$ the iteration (4.1) converges to the global solution in $H_{l, m}$.

Q.E.D.

Remark 1. While typing this work we received an abstract of Prof. Ukai that contains the existence theorem of the global solutions for the Cauchy problem of the nonlinear Boltzmann equation for the hard sphere molecule with the small initial data having no fluid part.

Remark 2. We can get the global solution of $(1 \cdot 1),(1 \cdot 2)$ without the restriction $f_{0} \in L^{1}\left(x ; L^{2}(v)\right)$. To prove this remark, we have only to note the facts

$$
\left\|e^{t B} f_{0}\right\|_{l} \leqq c\left\|f_{0}\right\|_{l} \leqq c\left\|f_{0}\right\|_{l, m}
$$

and $(4 \cdot 7)$ for $\beta=0$. Then the inequality (4.12) will become

$$
\|f\|_{0}^{l, m} \leqq a\left\|f_{0}\right\|_{l, m}+b\left(\|h\|_{0}^{l, m}\right)^{2} .
$$

Hence the iteration (4.1) gives the bounded (in $t$ ) solution in $H_{l, m}$, $l, m \geqq 3$.

Moreover if we note that in this case the $x$-derivatives of the solution have the decay order as

$$
\left\|\frac{\partial f(t)}{\partial x_{i}}\right\|_{2,3} \leqq \frac{c\left\|f_{0}\right\|_{3,3}}{(1+t)^{1 / 2}},\left\|\frac{\partial^{2} f(t)}{\partial x_{i} \partial x_{j}}\right\|_{1,3} \leqq \frac{c\left\|f_{0}\right\|_{3,3}}{(1+t)},
$$

we can get the decay of the solution $f(t)$ in the norm of $H_{0,3}$ by the aid of the Nirenberg's inequality(*);

$$
\|u(x)\|_{L^{\infty}} \leqq c\left(\sum_{i, j}\left\|\partial^{2} u / \partial x_{i} \partial x_{j}\right\|_{L^{2}}\right)^{3 / 4}\left(\|u\|_{L^{2}}\right)^{1 / 4}
$$

In fact we know that by the Lebesgue's theorem

$$
\left\|e^{t B} f_{0}\right\|_{0,3} \rightarrow 0 \quad \text { as } \quad t \rightarrow+\infty
$$

and we have for the nonlinear part

(*) L. Nirenberg, On elliptic partial differential equations, Annali Scuola Norm. Sup. Pisa, 13 (1959) 115-162. 


$$
\begin{aligned}
\int_{0}^{t}\left\|e^{(t-s) B} \nu \Gamma(f(s), f(s))\right\|_{H_{0}} d s \leqq \int_{0}^{t} \frac{c\|\nu \Gamma(s)\|_{H_{0}}}{(1+t-s)^{1 / 2}} d s \\
\quad \leqq \int_{0}^{t} \frac{c\|f(s)\|_{3,3} \cdot \sup _{v}(1+|v|)^{3}\|f(s)\|_{L^{\infty}}}{(1+t-s)^{1 / 2}} d s \\
\quad \leqq \int_{0}^{t} \frac{c\|f(s)\|_{3,3}^{5 / 4} \cdot\left(\sum\left\|\partial^{2} f(s) / \partial x_{i} \partial x_{j}\right\|_{0,3}\right)^{3 / 4}}{(1+t-s)^{1 / 2}} d s \\
\leqq \int_{0}^{t} c\left\|f_{0}\right\|_{3,3}^{2} d s /(1+t-s)^{1 / 2}(1+s)^{3 / 4} \\
\leqq c\left\|f_{0}\right\|_{3,3}^{2} /(1+t)^{1 / 4} .
\end{aligned}
$$

\section{References}

[1] Ellis, R. and Pinsky, M., The first and second fluid approximations to the linearized Boltzman equation, J. Math. Pures Appl., 54 (1975), 125-156.

[2] Grad, H., Asymptotic theory of the Boltzmann equation, II, Rarefied Gas Dynamics, J. A. Laurmann, ed. Vol. I, Academic Press, New York, 1963.

[3] — Asymptotic equivalence of the Navier-Stokes and nonlinear Boltzmann equations, Proc. Symp. in Appl. Math., Amer. Math. Soc., 17 (1965), 154-183.

[4] Solutions of the Boltzmann equation in an unbounded domain, Comm. Pure Appl. Math., 18 (1965), 345-354.

[5] Inoue, K. and Nishida, T., On the Broadwell model of the Boltzmann equation for a simple discrete velocity gas, to appear in Appl. Math. and Optimization (Intern. J.).

[6] Kato, T., Perturbation Theory for Linear Operators, Springer Verlag, New York, 1966.

[7] McLennan, J., Convergence of the Chapman-Enskog expansion for the linearized Boltzmann equation, Phys. of Fluids, 8 (1965), 1580-1584.

[8] Nicolaenco, B., Dispersion laws for plane wave propagation and the Boltzmann equation, Seminar on the Boltzmann Equation, Courant Inst. Math. Sci., NYU, F. Grünbaum, ed. (1972).

[9] Scharf, G., Functional-analytic discussion of the linearized Boltzmann equation, Helv. Phys. Acta, 40 (1967), 929-945.

[10] - Normal solutions of the linearized Boltzmann equation, Helv. Phys. Acta, 42 (1969), 5-22.

[11] Schechter, M., On the essential spectrum of an arbitrary operator I, J. Math. Anal. Appl., 13 (1966), 205-215.

[12] Ukai, S., On the existence of global solutions of mixed problem for nonlinear Boltzmann equation, Proc. Japan Acad., 50 (1974), 179-184.

[13] Arseniev, A., The Cauchy problem for the linearized Boltzman equation, J. Comput. Math. and Math. Phps. (USSR), 5 (1965), 864-882. 
